\title{
New homoclinic solutions for a class of second-order Hamiltonian systems with a mixed condition
}

\section{Xuefeng $\mathrm{Li}^{1}$ and Jin $\mathrm{Jia}^{1 *}$}

\section{${ }^{\text {*Correspondence: }}$}

judgecool@swufe.edu.cn

${ }^{1}$ Western China Center for

Economic Research, Southwestern

University of Finance and

Economics, Chengdu, China

\section{然 Springer}

\begin{abstract}
In this paper, we introduce a new mixed condition to obtain a new compact embedding theorem. Under this theorem, we study the existence and multiplicity of nontrivial homoclinic solutions for a class of second-order Hamiltonian systems with variable separated type nonlinear terms.
\end{abstract}

MSC: $34 \mathrm{C} 37 ; 37 \mathrm{~J} 45 ; 47 \mathrm{~J} 30$

Keywords: Homoclinic solutions; Second-order Hamiltonian systems; Mixed condition; Embedding theorem; Variational methods

\section{Introduction and main results}

In this paper, we consider the following second-order Hamiltonian system:

$$
\ddot{u}(t)-L(t) u+\nabla W(t, u)=0,
$$

where $W: \mathbb{R} \times \mathbb{R}^{N} \rightarrow \mathbb{R}$ is a $C^{1}$-map and $\nabla W(t, x)$ denotes the gradient with respect to the $x$ variable. $L \in C\left(\mathbb{R}, \mathbb{R}^{N^{2}}\right)$ is a matrix-valued function. A solution $u(t)$ of problem (1) is nontrivial homoclinic (to 0 ) if $u \neq \equiv, u(t) \rightarrow 0$ as $t \rightarrow \pm \infty$.

In the last two decades, many mathematicians have successfully used variational methods to obtain the existence and multiplicity of homoclinic orbits for problem (1) such as $[1,3-18,20-37]$. Since this problem is considered in the whole space, one of the main difficulties is the lack of compactness of embedding. In 1990, Rabinowitz [20] considered the periodic case. In his paper, $L(t)$ and $W(t, u)$ were assumed to be periodic in $t$ and the author obtained the results by the mountain pass theorem. Without the periodic condition, Rabinowitz and Tanaka [21] introduced the following coercive condition on $L(t)$.

$\left(L^{\prime}\right) L \in C\left(\mathbb{R}, \mathbb{R}^{N^{2}}\right)$ is a symmetric and positively definite matrix for all $t \in \mathbb{R}$, and there exists a continuous function $l: \mathbb{R} \rightarrow \mathbb{R}^{+}$satisfying

$$
(L(t) x, x) \geq l(t)|x|^{2} \quad \text { with } l(t) \rightarrow \infty \text { as }|t| \rightarrow \infty .
$$

Using $\left(L^{\prime}\right)$, many mathematicians studied problem (1) with superquadratic, subquadratic, or asymptotic quadratic nonlinearities. In this paper, we mainly consider the superquadratic growth case. First, we recall some main results.

(c) The Author(s) 2018. This article is distributed under the terms of the Creative Commons Attribution 4.0 International License (http://creativecommons.org/licenses/by/4.0/), which permits unrestricted use, distribution, and reproduction in any medium, provided you give appropriate credit to the original author(s) and the source, provide a link to the Creative Commons license, and indicate if changes were made. 
In 1991, Rabinowitz and Tanaka [21] used the so-called Ambrosetti-Rabinowitz ((AR) for short) condition to obtain the existence of homoclinic solutions for problem (1).

$(A R)$ There exists a constant $\theta>2$ such that

$$
0<\theta W(t, x) \leq(\nabla W(t, x), x)
$$

for every $t \in \mathbb{R}$ and $x \in \mathbb{R}^{N} \backslash\{0\}$.

Condition $(A R)$ is useful in guaranteeing the geometrical structure of the corresponding functional and the boundedness of Palais-Smale ((PS) for short) sequence. After then, there have been many mathematicians trying to weaken this condition. In 2009, Ding and Lee [6] introduced the following generalized superquadratic condition.

$\left(H_{1}\right)$ There exist $\epsilon \in(0,1)$ and $\kappa_{1}, b_{0}>0$ such that

$$
\widetilde{W}(t, x) \geq b_{0} \frac{(\nabla W(t, x), x)}{|x|^{2-\epsilon}} \quad \text { for all } t \in \mathbb{R} \text { and }|x| \geq \kappa_{1} \text {, }
$$

where

$$
\widetilde{W}(t, x)=(\nabla W(t, x), x)-2 W(t, x)
$$

In a recent paper, $\left(H_{1}\right)$ was weakened by Wu et al. [28] with the following condition.

$\left(H_{2}\right) \frac{\widetilde{W}(t, x)}{W(t, x)}|x|^{2} \rightarrow+\infty$ as $|x| \rightarrow \infty$ uniformly in $t \in \mathbb{R}$.

In 2011, Yang and Han [31] also made a contribution to the multiplicity of homoclinic solutions for problem (1) under the following monotonous condition.

$\left(H_{3}\right) s^{-1}(\nabla W(t, s x), x)$ is an increasing function of $s \in(0,1]$ for all $(t, x) \in \mathbb{R} \times \mathbb{R}^{N}$.

In 2013, Lv and Tang [15] showed that the following condition is weaker than $\left(H_{3}\right)$.

$\left(H_{4}\right)$ There exists $\mu \geq 1$ such that

$$
\mu \widetilde{W}(t, x) \geq \widetilde{W}(t, s x)
$$

for all $(t, x) \in \mathbb{R} \times \mathbb{R}^{N}$ and $s \in[0,1]$.

Conditions $\left(H_{1}\right)-\left(H_{4}\right)$ can be seen as the generalizations or supplements of $(A R)$. However, each result in the above papers implies that

$\left(H_{5}\right)$ There exists $\kappa_{2}>0$ such that $\sup _{t \in \mathbb{R},|x|=\kappa_{2}} W(t, x)<+\infty$.

Obviously, $\left(H_{5}\right)$ holds when $W(t, x)$ is periodic in $t$. If there is no periodic assumption, $\left(H_{5}\right)$ is an important requirement in many papers [1, 4-13, 16-18, 20, 21, 24, 27, 33, 35]. Recently, Yuan and Zhang [35] considered the following variable separation nonlinear term:

$$
W(t, x)=a(t) G(x)
$$

where $a: \mathbb{R} \rightarrow \mathbb{R}^{+}$is continuous, $G \in C^{1}\left(\mathbb{R}^{N}, \mathbb{R}\right)$. In [35], the authors considered the following conditions:

(A) $a(t) \rightarrow 0$ as $|t| \rightarrow \infty$;

$\left(L^{\prime \prime}\right) L \in C\left(\mathbb{R}, \mathbb{R}^{N^{2}}\right)$ is a symmetric and positively definite matrix for all $t \in \mathbb{R}$, and there exists a constant $M_{0}>0$ such that

$$
(L(t) x, x) \geq M_{0}|x|^{2} \quad \text { for all }(t, x) \in \mathbb{R} \times \mathbb{R}^{N} .
$$


Obviously, $\left(L^{\prime \prime}\right)$ is weaker than $\left(L^{\prime}\right)$. In a recent paper, Wu et al. [28] considered a class of fractional Hamiltonian systems with variable separated type nonlinear terms. They introduced the following condition.

$\left(L^{\prime \prime \prime}\right) \frac{a(t)}{l(t)} \rightarrow 0$ as $|t| \rightarrow \infty$, where $l(t) \in C(\mathbb{R}, \mathbb{R})$ satisfies

$$
l(t) \equiv \inf _{|x|=1}(L(t) x, x)
$$

Motivated by the above papers, in this paper, we introduce the following condition.

$\left(L_{1}\right)$ There exists a constant $r_{0}>0$ such that

$$
\lim _{|s| \rightarrow+\infty} \operatorname{meas}\left(\left\{t \in B_{r_{0}}(s): \frac{l(t)}{a(t)} \nsucceq M\right\}\right)=0, \quad \forall M>0,
$$

where $B_{r_{0}}(s)=\left\{t \in \mathbb{R}: s-r_{0} \leq t \leq s+r_{0}\right\}, l(t) \in C(\mathbb{R}, \mathbb{R})$ and meas $(\cdot)$ denotes the Lebesgue measure.

In [35], the nonlinear term $G(x)$ is assumed to satisfy the following classical $A R$-type condition:

$\left(A R^{\prime}\right)$ There exists a constant $v>2$ such that

$$
0<v G(x) \leq(\nabla G(x), x)
$$

for every $x \in \mathbb{R}^{N} \backslash\{0\}$.

From the $\left(A R^{\prime}\right)$ condition, we can deduce that

$(S Q) \widetilde{G}(x) \geq 0$ for any $x \in \mathbb{R}^{N}$, where $\widetilde{G}(x)=(\nabla G(x), x)-2 G(x)$.

In [28], the authors considered the following weakened superquadratic growth condition.

$\left(H_{6}\right)$ There exist $v>2$ and $b_{2}, \kappa_{3}>0$ such that

$$
(\nabla G(x), x)-v G(x) \geq-b_{2}|x|^{2} \quad \text { for all }|x| \geq \kappa_{3} .
$$

$\left(H_{6}\right)$ is a generalization of condition $\left(A R^{\prime}\right)$, which is only a local condition. Instead of $\left(H_{6}\right)$, the authors in [28] also considered the following superquadratic condition.

$\left(H_{7}\right) \frac{\widetilde{G}(x)}{G(x)}|x|^{2-r_{0}} \rightarrow+\infty$ as $|x| \rightarrow \infty$, where $r_{0}$ is the growth exponent of the subquadratic term at infinity with respect to $x$.

In this paper, we consider $G(x)$ satisfying the following monotonous condition.

$\left(W_{1}\right)$ There exists a constant $\theta \geq 1$ such that $\theta \widetilde{G}(x) \geq \widetilde{G}(s x)$ for all $x \in \mathbb{R}^{N}$ and $s \in[0,1]$.

Now we state our main results.

Theorem 1.1 Suppose (2), $\left(L^{\prime \prime}\right),\left(L_{1}\right),\left(W_{1}\right)$ and the following conditions hold:

$\left(L_{2}\right)$ There exists $A>0$ such that $a(t) \leq A l(t)$ for all $t \in \mathbb{R}$;

$\left(W_{2}\right) a(t) \in C\left(\mathbb{R}, \mathbb{R}^{+}\right)$

$\left(W_{3}\right) G(0)=0$ and $\nabla G(x)=o(|x|)$ as $|x| \rightarrow 0$;

$\left(W_{4}\right) G(x) /|x|^{2} \rightarrow+\infty$ as $|x| \rightarrow \infty$.

$\left(W_{5}\right)$ There are constants $\zeta>2$ and $d_{1}>0$ such that

$$
|G(x)| \leq d_{1}\left(|x|^{2}+|x|^{\zeta}\right) \quad \text { for all } x \in \mathbb{R}^{N} .
$$


Then problem (1) possesses at least one nontrivial homoclinic solution.

If $G(t)$ is even in $t$, we can obtain the following multiplicity result.

Theorem 1.2 Suppose (2), $\left(L^{\prime \prime}\right),\left(L_{1}\right),\left(L_{2}\right),\left(W_{1}\right)-\left(W_{5}\right)$ and $G(-x)=G(x)$ for all $x \in \mathbb{R}^{N}$. Then problem (1) possesses infinitely many solutions.

By a condition similar to $\left(H_{2}\right)$, we obtain the following.

Theorem 1.3 Suppose (2), $\left(L^{\prime \prime}\right),\left(L_{1}\right),\left(L_{2}\right),\left(W_{2}\right)-\left(W_{4}\right),(S Q)$ and the following condition holds:

$\left(W_{6}\right) \frac{\widetilde{G}(x)}{G(x)}|x|^{2} \rightarrow+\infty$ as $|x| \rightarrow \infty$.

Then problem (1) possesses at least one nontrivial homoclinic solution.

Theorem 1.4 Suppose (2), $\left(L^{\prime \prime}\right),\left(L_{1}\right),\left(L_{2}\right),\left(W_{2}\right)-\left(W_{4}\right),\left(W_{6}\right),(S Q)$ and $G(-x)=G(x)$ for all $x \in \mathbb{R}^{N}$. Then problem (1) possesses infinitely many solutions.

Remark 1 It can be seen that $a(t)$ and $l(t)$ are positive in our theorems and the results in [28]. Then we can deduce that $\left(L_{1}\right)$ is weaker than $\left(L^{\prime \prime \prime}\right)$. The reader can see [2] for more details.

Remark 2 Condition $\left(H_{5}\right)$ does not hold in our theorems since $a(t)$ can go to infinity as $|t| \rightarrow \infty$.

Remark 3 Condition $\left(W_{1}\right)$ implies $(S Q)$.

\section{Preliminaries}

Set

$$
E:=\left\{u \in H^{1}\left(\mathbb{R}, \mathbb{R}^{N}\right): \int_{\mathbb{R}}\left(|\dot{u}(t)|^{2}+(L(t) u(t), u(t))\right) d t<\infty\right\}
$$

with the inner product

$$
(u, v)_{E}=\int_{\mathbb{R}}((\dot{u}(t), \dot{v}(t))+(L(t) u(t), u(t))) d t .
$$

Let $L_{a}^{2}\left(\mathbb{R}, \mathbb{R}^{N}\right)$ be the weighted space of measurable functions $u: \mathbb{R} \rightarrow \mathbb{R}^{N}$ under the norm

$$
\|u\|_{L_{a}^{2}}=\left(\int_{\mathbb{R}} a(t)|u(t)|^{2} d t\right)^{1 / 2} .
$$

Throughout this paper, $C>0$ denotes a universal positive constant. Note that under condition $\left(L_{1}\right)$

$$
E \subset H^{1}\left(\mathbb{R}, \mathbb{R}^{N}\right) \subset L^{p}\left(\mathbb{R}, \mathbb{R}^{N}\right)
$$

for any $p \in[2,+\infty]$ with the embedding being continuous, which implies that there is a positive constant $C_{\infty}>0$ such that the following inequality holds:

$$
\max \left\{\|u\|_{2},\|u\|_{\infty}\right\} \leq C_{\infty}\|u\|
$$


First, we show a compact embedding theorem.

Lemma 2.1 Under conditions $\left(L_{1}\right)-\left(L_{2}\right)$ and $\left(L^{\prime \prime}\right)$, the embedding $E \hookrightarrow L_{a}^{2}\left(\mathbb{R}, \mathbb{R}^{N}\right)$ is continuous and compact.

Proof Suppose that $\left\{u_{n}\right\}$ is a bounded sequence in $E$. Then there exists $M_{0}>0$ such that $\left\|u_{n}\right\| \leq M_{0}$. Hence there exists a weak convergent subsequence, still denoted by $\left\{u_{n}\right\}$, such that $u_{n} \rightarrow u_{0}$ in $E$. Assuming $v_{n}=u_{n}-u_{0}$, we obtain that $\left\{v_{n}\right\}$ is a bounded sequence in $E$ and $v_{n} \rightarrow 0$ in $E$. Next, we show that $v_{n} \rightarrow 0$ in $L_{a}^{2}\left(\mathbb{R}, \mathbb{R}^{N}\right)$. It follows from the Sobolev compact embedding theorem that $v_{n} \rightarrow 0$ in $L^{1}\left(B_{R}(0), \mathbb{R}^{N}\right)$ for any $R>0$. Choose $\left\{s_{i}\right\} \subset \mathbb{R}$ such that $\mathbb{R} \subset \bigcup_{i=1}^{\infty} B_{r_{0}}\left(s_{i}\right)$ and each $t \in \mathbb{R}$ is contained by two such intervals at most. Set

$$
A(M, R)=\left\{t \in B_{R}^{c}(0): \frac{l(t)}{a(t)} \nsucceq M\right\}
$$

and

$$
B(M, R)=\left\{t \in B_{R}^{c}(0): \frac{l(t)}{a(t)} \geq M\right\} .
$$

On the one hand, we have

$$
\int_{B(M, R)} a(t)\left|v_{n}(t)\right|^{2} d t \leq \frac{1}{M} \int_{B(M, R)} l(t)\left|v_{n}(t)\right|^{2} d t \leq \frac{\left\|v_{n}\right\|^{2}}{M} \leq \frac{2 M_{0}}{M}
$$

On the other hand, let $\varepsilon_{R}=\sup _{s_{i}}\left(\operatorname{meas}\left(A(M, R) \bigcap B_{r_{0}}\left(s_{i}\right)\right)\right)$, we obtain

$$
\begin{aligned}
\int_{A(M, R)} a(t)\left|v_{n}(t)\right|^{2} d t & \leq \sum_{i=1}^{\infty} \int_{A(M, R) \cap B_{r_{0}}\left(s_{i}\right)} a(t)\left|v_{n}(t)\right|^{2} d t \\
& \leq \varepsilon_{R}^{\frac{1}{2}} \sum_{i=1}^{\infty}\left(\int_{A(M, R) \cap B_{r_{0}}\left(s_{i}\right)} a^{2}(t)\left|v_{n}(t)\right|^{4} d t\right)^{\frac{1}{2}} \\
& \leq A \varepsilon_{R}^{\frac{1}{2}} \sum_{i=1}^{\infty}\left(\int_{A(M, R) \cap B_{r_{0}}\left(s_{i}\right)} l^{2}(t)\left|v_{n}(t)\right|^{4} d t\right)^{\frac{1}{2}} \\
& \leq A C \varepsilon_{R}^{\frac{1}{2}} \sum_{i=1}^{\infty} \int_{B_{r_{0}}\left(s_{i}\right)}\left(|\dot{u}(t)|^{2}+(L(t) u(t), u(t))\right) d t \\
& \leq 2 A C \varepsilon_{R}^{\frac{1}{2}}\|u\|^{2}
\end{aligned}
$$

for some $C>0$. It follows from $\left(L_{2}\right)$ that $\int_{A(M, R)} a(t)\left|v_{n}(t)\right|^{2} d t \rightarrow 0$ as $R \rightarrow \infty$, which implies that

$$
\begin{aligned}
\int_{B_{R}^{c}(0)} a(t)\left|v_{n}(t)\right|^{2} d t & =\int_{A(M, R)} a(t)\left|v_{n}(t)\right|^{2} d t+\int_{B(M, R)} a(t)\left|v_{n}(t)\right|^{2} d t \\
& \leq \frac{2 M_{0}}{M}+2 d_{0} C \varepsilon_{R}^{\frac{1}{2}}\|u\|^{2} \\
& \rightarrow 0 \text { as } \min \{M, R\} \rightarrow \infty
\end{aligned}
$$


Then we can deduce that $v_{n} \rightarrow 0$ in $L_{a}^{2}\left(\mathbb{R}, \mathbb{R}^{N}\right)$. Hence the embedding $E \hookrightarrow L_{a}^{2}\left(\mathbb{R}, \mathbb{R}^{N}\right)$ is continuous and compact.

From Lemma 2.1, we can easily deduce that there is a positive constant $K>0$ such that the following inequality holds:

$$
\|u\|_{L_{a}^{2}} \leq K\|u\|
$$

Lemma 2.2 Suppose that conditions $\left(L^{\prime \prime}\right),(2),\left(L_{1}\right)$, and $\left(W_{4}\right)$ hold, then we have $\nabla G\left(u_{k}\right) \rightarrow$ $\nabla G(u)$ in $L_{a}^{2}\left(\mathbb{R}, \mathbb{R}^{N}\right)$ if $u_{k} \rightarrow u$ in $E$.

Proof The proof of this lemma is similar to that of Lemma 2.3 in [28].

The corresponding functional of (1) is defined by

$$
I(u)=\frac{1}{2}\|u\|^{2}-\int_{\mathbb{R}} W(t, u(t)) d t .
$$

Lemma 2.3 Suppose $\left(L^{\prime \prime}\right),(2),\left(L_{1}\right)$, and $\left(W_{4}\right)$ hold, then $I \in C^{1}(E, \mathbb{R})$ is w.l.s.c. and

$$
\left.\left\langle I^{\prime}(u), v\right\rangle=\int_{\mathbb{R}}((\dot{u}(t), \dot{v}(t))+(L(t) u(t), v(t))) d t-\int_{\mathbb{R}}(\nabla W(t, u(t)), v(t))\right) d t \quad \forall u, v \in E .
$$

Proof The proof is similar to Lemma 2.4 in [28].

From Lemma 2.3, we obtain

$$
\left\langle I^{\prime}(u), u\right\rangle=\|u\|^{2}-\int_{\mathbb{R}}(\nabla W(t, u(t)), u(t)) d t
$$

The following theorem is useful in proving the multiplicity of homoclinic solutions for problem (1).

Lemma 2.4 (see [19], Theorem 9.12) Let $\mathcal{B}$ be an infinite dimensional real Banach space, and let $I \in C^{1}(\mathcal{B}, \mathbb{R})$ be even, satisfy the $(P S)$ condition and $I(0)=0$. If $\mathcal{B}=V \oplus X$, where $V$ is finite dimensional and I satisfies the following:

$\left(A_{1}\right)$ There are constants $\varrho, \alpha>0$ such that $\left.I\right|_{\partial B_{e} \cap X} \geq \alpha$; and

$\left(A_{2}\right)$ For each finite dimensional subspace $\widetilde{E} \subset \mathcal{B}$, there is $r=r(\widetilde{E})$ such that $I \leq 0$ on $\widetilde{E} \backslash$ $B_{r(\widetilde{E})}$.

Then I has an unbounded sequence of critical values.

\section{Proof of Theorem 1.1}

The proof of Theorem 1.1 is divided into several lemmas.

Lemma 3.1 Suppose that $(S Q)$ and $\left(W_{3}\right)$ hold, then $G(x) \geq 0$ for all $x \in \mathbb{R}$.

Proof The proof of this lemma is similar to that of Lemma 7 in [15]. 
Definition 3.1 A sequence $\left\{u_{n}\right\} \subset X$, where $X$ is a real Banach space, is called a Cerami $\left((C)\right.$ for short) sequence of functional $I$ if $\left\{I\left(u_{n}\right)\right\}$ is bounded and $\left\|I^{\prime}\left(u_{n}\right)\right\|\left(1+\left\|u_{n}\right\|\right) \rightarrow 0$ as $n \rightarrow \infty$.

Definition 3.2 A functional $I$ is said to satisfy the (C) condition if any (C) sequence of $I$ possesses a convergent subsequence.

Lemma 3.2 Suppose that the conditions of Theorem 1.1 hold, then I satisfies the $(C)$ condition.

Proof Assume that $\left\{u_{n}\right\} \subset E$ is a sequence such that $\left\{I\left(u_{n}\right)\right\}$ is bounded and $\left\|I^{\prime}\left(u_{n}\right)\right\|(1+$ $\left.\left\|u_{n}\right\|\right) \rightarrow 0$ as $n \rightarrow \infty$. Then, for some $M_{1}>0$, it follows

$$
\left|I\left(u_{n}\right)\right| \leq M_{1}, \quad\left\|I^{\prime}\left(u_{n}\right)\right\|\left(1+\left\|u_{n}\right\|\right) \leq M_{1} .
$$

Next we show that $\left\{u_{n}\right\}$ is bounded in $E$. Assuming $\left\|u_{n}\right\| \rightarrow+\infty$ as $n \rightarrow \infty$, set $w_{n}=\frac{u_{n}}{\left\|u_{n}\right\|}$, then $\left\|w_{n}\right\|=1$, which implies that there exists a subsequence of $\left\{w_{n}\right\}$, still denoted by $\left\{w_{n}\right\}$, such that $w_{n} \rightarrow w_{0}$ in $E$. By (7) and (9), we get

$$
\left|\int_{\mathbb{R}} \frac{W\left(t, u_{n}(t)\right)}{\left\|u_{n}\right\|^{2}} d t-\frac{1}{2}\right|=\left|-\frac{I\left(u_{n}\right)}{\left\|u_{n}\right\|^{2}}\right| \leq \frac{M_{1}}{\left\|u_{n}\right\|^{2}},
$$

which implies that

$$
\left|\int_{\mathbb{R}} \frac{W\left(t, u_{n}(t)\right)}{\left\|u_{n}\right\|^{2}} d t\right| \leq 1
$$

for $n$ large enough. The following discussion is divided into two cases.

Case 1: $w_{0} \not \equiv 0$. Let $\Omega=\left\{t \in \mathbb{R}|| w_{0}(t) \mid>0\right\}$. We can see that meas $(\Omega)>0$. Then there exists $\chi>0$ such that meas $(\Lambda)>0$, where $\Lambda=\Omega \bigcap B_{\chi}(0)$. Since $\left\|u_{n}\right\| \rightarrow+\infty$ as $n \rightarrow \infty$ and $\left|u_{n}(t)\right|=\left|w_{n}(t)\right| \cdot\left\|u_{n}\right\|$, we have $\left|u_{n}(t)\right| \rightarrow+\infty$ as $n \rightarrow \infty$ for a.e. $t \in \Lambda$. Let $a_{1}=\inf _{t \in B_{\chi}(0)} a(t)>0$. By $(2),\left(W_{2}\right),\left(W_{4}\right)$, Lemma 3.1, and Fatou's lemma, we can obtain

$$
\begin{aligned}
\lim _{n \rightarrow \infty} \int_{\mathbb{R}} \frac{a(t) G\left(u_{n}(t)\right)}{\left\|u_{n}\right\|^{2}} d t & \geq a_{1} \lim _{n \rightarrow \infty} \int_{\Lambda} \frac{G\left(u_{n}(t)\right)}{\left|u_{n}(t)\right|^{2}}\left|w_{n}(t)\right|^{2} d t \\
& \geq a_{1} \lim _{n \rightarrow \infty} \int_{\Lambda} \frac{G\left(u_{n}(t)\right)}{\left|u_{n}(t)\right|^{2}}\left|w_{n}(t)\right|^{2} d t \\
& \geq a_{1} \lim _{n \rightarrow \infty} \int_{\Lambda} \frac{G\left(u_{n}(t)\right)}{\left|u_{n}(t)\right|^{2}}\left|w_{n}(t)\right|^{2} d t \\
& =+\infty,
\end{aligned}
$$

which contradicts (11).

Case 2: $w_{0} \equiv 0$. Set a sequence $\left\{T_{n}\right\} \subset[0,1]$ such that $I\left(T_{n} u_{n}\right)=\max _{T \in[0,1]} I\left(T u_{n}\right)$. By Lemma 3.1, $\left(W_{2}\right),\left(W_{5}\right)$, and Lemma 2.1 , we obtain

$$
\begin{aligned}
0 & \leq \int_{\mathbb{R}} a(t) G\left(4 \sqrt{\theta M_{1}} w_{n}(t)\right) d t \\
& \leq d_{1}\left(16 \theta M_{1} \int_{\mathbb{R}} a(t)\left|w_{n}(t)\right|^{2} d t+\left(4 \sqrt{\theta M_{1}}\right)^{\zeta} \int_{\mathbb{R}} a(t)\left|w_{n}(t)\right|^{\zeta} d t\right)
\end{aligned}
$$




$$
\begin{aligned}
& =d_{1}\left(16 \theta M_{1}+\left(4 \sqrt{\theta M_{1}}\right)^{\zeta}\left\|w_{n}\right\|_{\infty}^{\zeta-2}\right) \int_{\mathbb{R}} a(t)\left|w_{n}(t)\right|^{2} d t \\
& =d_{1}\left(16 \theta M_{1}+\left(4 \sqrt{\theta M_{1}}\right)^{\zeta} C_{\infty}^{\zeta-2}\right) \int_{\mathbb{R}} a(t)\left|w_{n}(t)\right|^{2} d t \rightarrow 0 \quad \text { as } n \rightarrow \infty
\end{aligned}
$$

which implies that

$$
\int_{\mathbb{R}} a(t) G\left(4 \sqrt{\theta M_{1}} w_{n}(t)\right) d t \rightarrow 0 \quad \text { as } n \rightarrow \infty
$$

By the definition of $T_{n}$ and (12), for $n$ large enough, we have

$$
\begin{aligned}
I\left(T_{n} u_{n}\right) & \geq I\left(\frac{4 \sqrt{\theta M_{1}}}{\left\|u_{n}\right\|} u_{n}\right) \\
& =I\left(4 \sqrt{\theta M_{1}} w_{n}\right) \\
& =\frac{1}{2}\left\|4 \sqrt{\theta M_{1}} w_{n}\right\|^{2}-\int_{\mathbb{R}} a(t) G\left(4 \sqrt{\theta M_{1}} w_{n}(t)\right) d t \\
& =8 \theta M_{1}-\int_{\mathbb{R}} a(t) G\left(4 \sqrt{\theta M_{1}} w_{n}(t)\right) d t \\
& \geq 4 \theta M_{1} .
\end{aligned}
$$

Then we obtain

$$
\begin{aligned}
& \left\|T_{n} u_{n}\right\|^{2}-\int_{\mathbb{R}} a(t)\left(\nabla G\left(T_{n} u_{n}(t)\right), T_{n} u_{n}(t)\right) d t \\
& \quad=\left\langle I^{\prime}\left(T_{n} u_{n}(t)\right), T_{n} u_{n}(t)\right\rangle=\left.T_{n} \frac{d I\left(T u_{n}(t)\right)}{d T}\right|_{T=T_{n}}=0 .
\end{aligned}
$$

Hence, it follows from (13) and ( $\left.W_{1}\right)$ that

$$
\begin{aligned}
\int_{\mathbb{R}} a(t)\left(\frac{1}{2}\left(\nabla G\left(u_{n}(t)\right), u_{n}(t)\right)-G\left(u_{n}(t)\right)\right) d t \\
=\frac{1}{2} \int_{\mathbb{R}} a(t) \widetilde{G}\left(u_{n}(t)\right) d t \\
\quad \geq \frac{1}{2 \theta} \int_{\mathbb{R}} a(t) \widetilde{G}\left(T_{n} u_{n}(t)\right) d t \\
=\frac{1}{\theta} \int_{\mathbb{R}}\left(\frac{1}{2} a(t)\left(\nabla G\left(T_{n} u_{n}(t)\right), T_{n} u_{n}(t)\right)-a(t) G\left(T_{n} u_{n}(t)\right)\right) d t \\
=\frac{1}{\theta}\left(\frac{1}{2}\left\|T_{n} u_{n}\right\|^{2}-\int_{\mathbb{R}} a(t) G\left(T_{n} u_{n}(t)\right) d t\right) \\
=\frac{1}{\theta} I\left(T_{n} u_{n}\right),
\end{aligned}
$$

which implies that

$$
\int_{\mathbb{R}} a(t)\left(\frac{1}{2}\left(\nabla G\left(u_{n}(t)\right), u_{n}(t)\right)-G\left(u_{n}(t)\right)\right) d t \geq 4 M_{1} \quad \text { for } n \text { large enough. }
$$


However, we can deduce from (9) that

$$
\left|\int_{\mathbb{R}} a(t)\left(\frac{1}{2}\left(\nabla G\left(u_{n}(t)\right), u_{n}(t)\right)-G\left(u_{n}(t)\right)\right) d t\right|=\left|2 I\left(u_{n}\right)-\left\langle I^{\prime}\left(u_{n}\right), u_{n}\right\rangle\right| \leq 3 M_{1}
$$

for all $n \in \mathbb{N}$, which contradicts (14). Hence $\left\{u_{n}\right\}$ is bounded in $E$. Going if necessary to a subsequence, we can assume that $u_{n} \rightarrow u$ in $E$, which yields

$$
\left\langle I^{\prime}\left(u_{n}\right)-I^{\prime}(u), u_{n}-u\right\rangle \rightarrow 0 \quad \text { as } n \rightarrow \infty
$$

and it follows from Lemma 2.1, Hölder's inequality, (6), and Lemma 2.2 that

$$
\begin{aligned}
\left|\int_{\mathbb{R}} a(t)\left(\nabla G\left(u_{n}(t)\right)-\nabla G(u(t)), u_{n}(t)-u(t)\right) d t\right| & \leq\left\|\nabla G\left(u_{n}\right)-\nabla G(u)\right\|_{L_{a}^{2}}\left\|u_{n}-u\right\|_{L_{a}^{2}} \\
& \rightarrow 0 \quad \text { as } n \rightarrow \infty .
\end{aligned}
$$

Hence, we conclude that

$$
\begin{aligned}
& \left\|u_{n}-u\right\|^{2} \\
& \quad=\left\langle I^{\prime}\left(u_{n}\right)-I^{\prime}(u), u_{n}-u\right\rangle+\int_{\mathbb{R}} a(t)\left(\nabla G\left(u_{n}(t)\right)-\nabla G(u(t)), u_{n}(t)-u(t)\right) d t \\
& \quad \rightarrow 0 \quad \text { as } n \rightarrow \infty .
\end{aligned}
$$

We finish the proof.

Subsequently, we need the following lemmas to prove Theorem 1.1.

Lemma 3.3 Suppose that (2), $\left(L^{\prime \prime}\right),\left(L_{1}\right),\left(L_{2}\right),\left(W_{1}\right)$, and $\left(W_{3}\right)$ hold, then there exist constants $\varrho, \alpha>0$ such that $\left.I\right|_{\partial B_{\varrho}(0)} \geq \alpha$.

Proof By $\left(W_{3}\right)$, for any $\varepsilon>0$, there is $\sigma>0$ such that

$$
|G(x)| \leq \varepsilon|x|^{2}
$$

for all $|x| \leq \sigma$. For $\varepsilon_{1}=\frac{1}{4 A}$, there exists $\delta_{1}>0$ such that (15) holds. Set

$$
\varrho=C_{\infty}^{-1} \delta_{1}, \quad \alpha=\frac{1}{4} \varrho^{2}
$$

By (7), (15), (2), $\left(L_{2}\right)$, and $\left(W_{1}\right)$, for any $\|u\|=\varrho$, we obtain

$$
\begin{aligned}
I(u) & =\frac{1}{2}\|u\|^{2}-\int_{\mathbb{R}} W(t, u(t)) d t \\
& =\frac{1}{2}\|u\|^{2}-\int_{\mathbb{R}} a(t) G(u(t)) d t \\
& \geq \frac{1}{2}\|u\|^{2}-\frac{1}{4 A} \int_{\mathbb{R}} a(t)|u(t)|^{2} d t
\end{aligned}
$$




$$
\begin{aligned}
& \geq \frac{1}{2}\|u\|^{2}-\frac{1}{4} \int_{\mathbb{R}} l(t)|u(t)|^{2} d t \\
& \geq \frac{1}{4}\|u\|^{2} .
\end{aligned}
$$

By the definition of $\varrho$ and $\alpha$, (16) implies $\left.I\right|_{\partial B_{\varrho}(0)} \geq \alpha$.

Lemma 3.4 Suppose that (2), $\left(L^{\prime \prime}\right),\left(W_{2}\right)$, and $\left(W_{4}\right)$ hold, then there exists $\bar{e} \in E$ such that $\|\bar{e}\|>\varrho$ and $I(\bar{e}) \leq 0$, where $\varrho$ is defined in Lemma 3.3.

Proof Set $e_{0} \in C_{0}^{\infty}(-1,1)$ with $\left\|e_{0}\right\|=1$. Let $a_{2}=\min _{t \in B_{1}(0)} a(t)$ and $a_{3}=\max _{t \in B_{1}(0)} a(t)$. For $\beta>\frac{1}{2 a_{2} \int_{-1}^{1}\left|e_{0}(t)\right|^{2} d t}$, it follows from $\left(W_{4}\right)$ that there exists $\xi>0$ such that

$$
G(x) \geq \beta|x|^{2}
$$

for all $|x|>\xi$. By Lemma 3.1, we have

$$
G(x) \geq \beta\left(|x|^{2}-\xi^{2}\right)
$$

for all $x \in \mathbb{R}^{N}$. By (2), (7), and (17), for every $\eta \in \mathbb{R} \backslash\{0\}$, we have

$$
\begin{aligned}
I\left(\eta e_{0}\right) & =\frac{\eta^{2}}{2}\left\|e_{0}\right\|^{2}-\int_{-1}^{1} W\left(t, \eta e_{0}(t)\right) d t \\
& =\frac{\eta^{2}}{2}\left\|e_{0}\right\|^{2}-\int_{-1}^{1} a(t) G\left(\eta e_{0}(t)\right) d t \\
& \leq \frac{\eta^{2}}{2}-a_{2} \beta \eta^{2} \int_{-1}^{1}\left|e_{0}(t)\right|^{2} d t+2 a_{3} \beta \xi^{2} \\
& =\left(\frac{1}{2}-a_{2} \beta \int_{-1}^{1}\left|e_{0}(t)\right|^{2} d t\right) \eta^{2}+2 a_{3} \beta \xi^{2},
\end{aligned}
$$

which implies that

$$
I\left(\eta e_{0}\right) \rightarrow-\infty \quad \text { as } \eta \rightarrow+\infty
$$

Then there exists $\eta_{0} \in \mathbb{R} \backslash\{0\}$ such that $\left\|\eta_{0} e_{0}\right\|>\varrho$ and $I\left(\eta_{0} e_{0}\right)<0$. Letting $\bar{e}(t)=\eta_{0} e_{0}(t)$, we finish the proof.

Proof of Theorem 1.1 It is known that a deformation lemma can be proved when the usual $(P S)$ condition is replaced with condition $(C)$. From the above proofs and the mountain pass theorem (see [19], Theorem 2.2), I possesses a critical value $c \geq \alpha$ and a critical point $u_{0}$ such that $I\left(u_{0}\right)=c$, which means problem (1) has at least one nontrivial homoclinic solution.

\section{Proof of Theorem 1.3}

Lemma 4.1 Suppose that the conditions of Theorem 1.2 hold, then I satisfies the (C) condition. 
Proof Assuming that $\left\{u_{n}\right\} \subset E$ is a $(C)$ sequence of $I$, there exists a constant $M_{2}>0$ such that

$$
\left|I\left(u_{n}\right)\right| \leq M_{2}, \quad\left\|I^{\prime}\left(u_{n}\right)\right\|\left(1+\left\|u_{n}\right\|\right) \leq M_{2} .
$$

Now we show the boundedness of $\left\{u_{n}\right\}$. Arguing in an indirect way, we assume that $\left\|u_{n}\right\| \rightarrow+\infty$ as $n \rightarrow \infty$. Set $w_{n}=\frac{u_{n}}{\left\|u_{n}\right\|}$, then $\left\|w_{n}\right\|=1$, which implies that there exists a subsequence of $\left\{w_{n}\right\}$, still denoted by $\left\{w_{n}\right\}$, such that $w_{n} \rightarrow w_{0}$ in $E$. Similar to the proof of Lemma 3.2, we have

$$
\int_{\mathbb{R}} \frac{W\left(t, u_{n}\right)}{\left\|u_{n}\right\|^{2}} d t \rightarrow \frac{1}{2} \quad \text { as } n \rightarrow \infty
$$

Case 1: $w_{0} \not \equiv 0$. The proof is similar to the proof of Case 1 in Lemma 3.2.

Case 2: $w_{0} \equiv 0$. Let $\varepsilon=1$, then there exists $\sigma_{0}>0$ such that (15) holds for all $|x| \leq \sigma_{0}$. By $\left(W_{6}\right)$, we obtain that for any $B>0$, there exists $r_{\infty}>0$ such that, for all $|x| \geq r_{\infty}$, we have

$$
\frac{\widetilde{G}(x)}{G(x)}|x|^{2} \geq B
$$

It follows from (7), (8), (20), ( $\left.W_{1}\right)$, and (SQ) that

$$
\begin{aligned}
0 \leq & \int_{\mathbb{R}} \frac{W\left(t, u_{n}\right)}{\left\|u_{n}\right\|^{2}} d t \\
\leq & \int_{\left\{t \in \mathbb{R}|| u_{n} \mid>r_{\infty}\right\}} \frac{W\left(t, u_{n}\right)}{\left|u_{n}(t)\right|^{2}}\left|w_{n}(t)\right|^{2} d t+\int_{\left\{t \in \mathbb{R}|| u_{n} \mid \leq \sigma_{0}\right\}} \frac{W\left(t, u_{n}\right)}{\left\|u_{n}\right\|^{2}} d t \\
& +\int_{\left\{t \in \mathbb{R}\left|\sigma_{0} \leq\right| u_{n} \mid \leq r_{\infty}\right\}} \frac{W\left(t, u_{n}\right)}{\left\|u_{n}\right\|^{2}} d t \\
\leq & \left\|w_{n}\right\|_{L^{\infty}}^{2} \int_{\left\{t \in \mathbb{R}|| u_{n} \mid>r_{\infty}\right\}} \frac{a(t) G\left(u_{n}\right)}{\left|u_{n}(t)\right|^{2}} d t+\int_{\left\{t \in \mathbb{R} \|\left|u_{n}\right| \leq \sigma_{0}\right\}} a(t)\left|w_{n}(t)\right|^{2} d t \\
& +\int_{\left\{t \in \mathbb{R}\left|\sigma_{0} \leq\right| u_{n} \mid \leq r_{\infty}\right\}} \frac{a(t) G\left(u_{n}\right)\left|u_{n}(t)\right|^{2}}{\sigma_{0}^{2}\left\|u_{n}\right\|^{2}} d t \\
\leq & \frac{\left\|w_{n}\right\|_{L^{\infty}}^{2}}{B} \int_{\left\{t \in \mathbb{R} \|\left|u_{n}\right|>r_{\infty}\right\}} a(t)\left(\left(\nabla G\left(u_{n}\right), u_{n}\right)-2 G\left(u_{n}\right)\right) d t+\left\|w_{n}\right\|_{L_{a}^{2}}^{2} \\
& +\frac{\max _{\sigma_{0} \leq|x| \leq \rho_{\infty}}|G(x)|}{\sigma_{0}^{2}} \int_{\left\{t \in \mathbb{R}\left|\sigma_{0} \leq\right| u_{n} \mid \leq r_{\infty}\right\}} a(t)\left|w_{n}(t)\right|^{2} d t \\
\leq & \frac{\left\|w_{n}\right\|_{L^{\infty}}^{2}}{B} \int_{\left\{t \in \mathbb{R} \|\left|u_{n}\right|>r_{\infty}\right\}} a(t)\left(\left(\nabla G\left(u_{n}\right), u_{n}\right)-2 G\left(u_{n}\right)\right) d t \\
& +\left(1+\frac{\max _{\sigma_{0} \leq|x| \leq \rho_{\infty}}|G(x)|}{\sigma_{0}^{2}}\right)\left\|w_{n}\right\|_{L_{a}^{2}}^{2} \\
\leq & \frac{\left\|w_{n}\right\|_{L^{\infty}}^{2}}{B}\left(2 I\left(u_{n}\right)-\left\langle I^{\prime}\left(u_{n}\right), u_{n}\right\rangle\right)+\left(1+\frac{\max _{\sigma_{0} \leq|x| \leq \rho_{\infty}}|G(x)|}{\sigma_{0}^{2}}\right)\left\|w_{n}\right\|_{L_{a}^{2}}^{2} \\
\leq & \frac{3 M_{2} C_{\infty}^{2}}{B}+\left(1+\frac{\max _{\sigma_{0} \leq|x| \leq \rho_{\infty}}|G(x)|}{\sigma_{0}^{2}}\right)\left\|w_{n}\right\|_{L_{a}^{2}}^{2} . \\
&
\end{aligned}
$$


By the arbitrariness of $B$ and Lemma 3.1, we have

$$
\int_{\mathbb{R}} \frac{W\left(t, u_{n}\right)}{\left\|u_{n}\right\|^{2}} d t<\frac{1}{4}
$$

for $n$ large enough, which contradicts (19). Hence $\left\|u_{n}\right\|$ is bounded in $E$. Similar to the proof of Lemma 3.2, we see that $I$ satisfies the $(C)$ condition.

The following proof is similar to that of Theorem 1.1.

\section{Proof of Theorems 1.2 and 1.4}

As we know, Lemma 2.4 still holds when we replace the $(P S)$ condition with the $(C)$ condition. From Lemmas 3.2 and 4.1, we can see that $I$ satisfies the $(C)$ condition under the conditions of Theorems 1.2 and 1.4, respectively. Next, we only show that $I$ satisfies the geometric conditions of Lemma 2.4. Obviously, Lemma 3.3 holds under the conditions of Theorems 1.2 and 1.4, respectively. If we take $V=\{0\}$ and $X=E$, we can see there are constants $\varrho_{1}, \alpha_{1}>0$ such that $\left.I\right|_{\partial B_{\varrho_{1}} \cap X} \geq \alpha_{1}$ by Lemma 3.2, which means $\left(A_{1}\right)$ is fulfilled. Subsequently, we show that $I$ satisfies $\left(A_{2}\right)$.

Lemma 5.1 Suppose that (2), $\left(L^{\prime \prime}\right),\left(L_{1}\right),\left(L_{2}\right),\left(W_{3}\right)$, and $\left(W_{4}\right)$ hold, then I satisfies $\left(A_{2}\right)$.

Proof Let $\widetilde{E} \subset E$ be a finite dimensional subspace. For any $u \in \widetilde{E} \backslash\{0\}$ and $\vartheta>0$, set

$$
\Gamma_{\vartheta}(u)=\{t \in \mathbb{R}:|u(t)| \geq \vartheta\|u\|\}
$$

Similar to Lemma 6.2 in [29], there exists $\vartheta_{0}>0$ such that

$$
\operatorname{meas}\left(\Gamma_{\vartheta_{0}}(u)\right) \geq \vartheta_{0}
$$

for all $u \in \widetilde{E} \backslash\{0\}$. It is easy to see that there exists $\rho>0$ such that meas $\Upsilon_{\vartheta_{0}}(u)>\frac{1}{2} \vartheta_{0}$ for any $u \in \widetilde{E} \backslash\{0\}$, where $\Upsilon_{\vartheta_{0}}(u)=\Gamma_{\vartheta_{0}}(u) \bigcap B_{\rho}(0)$. Set $a_{4}=\inf _{|t| \leq \rho} a(t)>0$. By $\left(W_{4}\right)$, there exists $\gamma>0$ such that

$$
G(u(t)) \geq \frac{1}{a_{4} \vartheta_{0}^{3}}|u(t)|^{2} \geq \frac{1}{a_{4} \vartheta_{0}}\|u\|^{2}
$$

for all $u \in \widetilde{E}$ and $t \in \Upsilon_{\vartheta_{0}}(u)$ with $\|u\| \geq \gamma$. Then, for any $u \in \widetilde{E} \backslash B_{\gamma}$, it follows from (7), $\left(W_{1}\right),\left(W_{3}\right)$, and (23) that

$$
\begin{aligned}
I(u) & =\frac{1}{2}\|u\|^{2}-\int_{\mathbb{R}} a(t) G(u(t)) d t \\
& =\frac{1}{2}\|u\|^{2}-\int_{\Upsilon_{\vartheta_{0}}(u)} a(t) G(u(t)) d t-\int_{\mathbb{R} \backslash \Upsilon_{\vartheta_{0}}(u)} a(t) G(u(t)) d t \\
& \leq \frac{1}{2}\|u\|^{2}-a_{4} \int_{\Upsilon_{\vartheta_{0}}(u)} G(u(t)) d t \\
& \leq \frac{1}{2}\|u\|^{2}-\frac{1}{\vartheta_{0}} \operatorname{meas}\left(\Upsilon_{\vartheta_{0}}(u)\right)\|u\|^{2}
\end{aligned}
$$




$$
\begin{aligned}
& \leq \frac{1}{2}\|u\|^{2}-\|u\|^{2} \\
& =-\frac{1}{2}\|u\|^{2} .
\end{aligned}
$$

Then there exists $r>\gamma$ such that $\left.I\right|_{\tilde{E} \backslash B_{r}} \leq 0$.

Since $I$ is even in $u, I$ possesses an unbounded sequence of critical values by Lemma 2.4, which proves Theorems 1.2 and 1.4 .

\section{Acknowledgements}

Not applicable.

\section{Funding}

This work is supported by the National Natural Science Foundation of China, "The Research of Industrial Clusters on Rural Poverty Reduction from the Perspective of Spatial Econometrics" (Grant number 71503212).

\section{Abbreviations}

(AR) condition, Ambrosetti-Rabinowitz condition; (PS) sequence, Palais-Smale sequence; (C) condition, Cerami condition; (C) sequence, Cerami sequence.

\section{Availability of data and materials}

Data sharing not applicable to this article as no datasets were generated or analysed during the current study.

\section{Competing interests}

The authors declare that they have no competing interests.

\section{Authors' contributions}

The research and writing of this manuscript was a collaborative effort from all authors. $X \mathrm{~L}$ and JJ discussed it together, $\mathrm{XL}$ managed this manuscript, JJ revised it. All authors read and approved the final manuscript.

\section{Publisher's Note}

Springer Nature remains neutral with regard to jurisdictional claims in published maps and institutional affiliations.

Received: 9 June 2018 Accepted: 23 August 2018 Published online: 07 September 2018

\section{References}

1. Ambrosetti, A., Coti Zelati, V.: Multiple homoclinic orbits for a class of conservative systems. Rend. Semin. Mat. Univ. Padova 89, 177-194 (1993)

2. Bartsch, T., Wang, Z.Q.: Existence and multiplicity results for some superlinear elliptic problems on $\mathbf{R}^{N}$. Commun Partial Differ. Equ. 20(9-10), 1725-1741 (1995)

3. Carrião, P.C., Miyagaki, O.H.: Existence of homoclinic solutions for a class of time-dependent Hamiltonian systems. J. Math. Anal. Appl. 230, 157-172 (1999)

4. Chen, H.W., He, Z.M.: Infinitely many homoclinic solutions for a class of second-order Hamiltonian systems. Adv. Differ. Equ. 2014, 161 (2014)

5. Ding, Y.H.: Existence and multiplicity results for homoclinic solutions to a class of Hamiltonian systems. Nonlinear Anal. 25, 1095-1113 (1995)

6. Ding, Y.H., Lee, C.: Homoclinics for asymptotically quadratic and superquadratic Hamiltonian systems. Nonlinear Anal. 71, 1395-1413 (2009)

7. Ding, Y.H., Li, S.J.: Homoclinic orbits for first order Hamiltonian systems. J. Math. Anal. Appl. 189, 585-601 (1995)

8. Felmer, P.L., Silva, E.A.B.: Homoclinic and periodic orbits for Hamiltonian systems. Ann. Sc. Norm. Super. Pisa, Cl. Sci. 26, 285-301 (1998)

9. Izydorek, M., Janczewska, J.: Homoclinic solutions for a class of the second order Hamiltonian systems. J. Differ. Equ. 219, 375-389 (2005)

10. Izydorek, M., Janczewska, J.: Homoclinic solutions for nonautonomous second-order Hamiltonian systems with a coercive potential. J. Math. Anal. Appl. 335, 1119-1127 (2007)

11. Korman, P., Lazer, A.C.: Homoclinic orbits for a class of symmetric Hamiltonian systems. Electron. J. Differ. Equ. 1994, 1 , $1-10(1994)$

12. Lu, S.P.: Homoclinic solutions for a nonlinear second order differential system with $p$-Laplacian operator. Nonlinear Anal., Real World Appl. 12, 525-534 (2011)

13. LV, Y., Tang, C.-L.: Existence of even homoclinic orbits for second-order Hamiltonian systems. Nonlinear Anal. 67 2189-2198 (2007)

14. LV, Y., Tang, C.-L.: Homoclinic orbits for second-order Hamiltonian systems with subquadratic potentials. Chaos Solitons Fractals 57, 137-145 (2013)

15. LV, Y., Tang, C.-L.: Existence and multiplicity of homoclinic orbits for second-order Hamiltonian systems with superquadratic potential. Abstr. Appl. Anal 2013, 328630 (2013)

16. Omana, W., Willem, M.: Homoclinic orbits for a class of Hamiltonian systems. Differ. Integral Equ. 5, 1115-1120 (1992) 
17. Ou, Z.-Q., Tang, C.-L.: Existence of homoclinic solution for the second order Hamiltonian systems. J. Math. Anal. Appl. 291, 203-213 (2004)

18. Paturel, E.: Multiple homoclinic orbits for a class of Hamiltonian systems. Calc. Var. Partial Differ. Equ. 12, 117-143 (2001)

19. Rabinowitz, P.H.: Minimax methods in critical point theory with applications to differential equations. In: CBMS, Regional Conf. Ser. in Math., vol. 65. Am. Math. Soc., Providence (1986)

20. Rabinowitz, P.H.: Homoclinic orbits for a class of Hamiltonian systems. Proc. R. Soc. Edinb. A 114, 33-38 (1990)

21. Rabinowitz, P.H., Tanaka, K.: Some results on connecting orbits for a class of Hamiltonian systems. Math. Z. 206, 473-499 (1991)

22. Sun, J., Chen, H., Nieto, J.J.: Homoclinic solutions for a class of subquadratic second-order Hamiltonian systems. J. Math. Anal. Appl. 373, 20-29 (2011)

23. Sun, J., Chen, H., Nieto, J.J.: Homoclinic orbits for a class of first-order nonperiodic asymptotically quadratic Hamiltonian systems with spectrum point zero. J. Math. Anal. Appl. 378, 117-127 (2011)

24. Sun, J., Wu, T.F.: Multiplicity and concentration of homoclinic solutions for some second order Hamiltonian systems. Nonlinear Anal. 114, 105-115 (2015)

25. Sun, J., Wu, T.F.: Homoclinic solutions for a second-order Hamiltonian system with a positive semi-definite matrix. Chaos Solitons Fractals 76, 24-31 (2015)

26. Tang, X.H., Lin, X.Y.: Infinitely many homoclinic orbits for Hamiltonian systems with indefinite sign subquadratic potentials. Nonlinear Anal. 74, 6314-6325 (2011)

27. Tang, X.H., Xiao, L.: Homoclinic solutions for a class of second order Hamiltonian systems. Nonlinear Anal. 71, 1140-1152 (2009)

28. Wu, D.-L., Li, C., Yuan, P.F.: Multiplicity solutions for a class of fractional Hamiltonian systems with concave-convex potentials. Mediterr. J. Math. 15, 35 (2018)

29. Wu, D.-L., Tang, C.-L., Wu, X.-P.. Homoclinic orbits for a class of second-order Hamiltonian systems with concave-convex nonlinearities. Electron. J. Qual. Theory Differ. Equ. 2018, 6, 1-18 (2018)

30. Yang, L., Chen, H., Sun, J.: Infinitely many homoclinic solutions for some second order Hamiltonian systems. Nonlinear Anal. 74, 6459-6468 (2011)

31. Yang, M.-H., Han, Z.-Q.: Infinitely many homoclinic solutions for second-order Hamiltonian systems with odd nonlinearities. Nonlinear Anal. 74, 2635-2646 (2011)

32. Yang, M.-H., Han, Z.-Q.: Infinitely many homoclinic solutions for second-order Hamiltonian systems with odd nonlinearities. Nonlinear Anal. 74, 2635-2646 (2011)

33. Ye, Y.-W., Tang, C.-L:: Multiple homoclinic solutions for second-order perturbed Hamiltonian systems. Stud. Appl. Math. 132, 112-137 (2014)

34. Ye, Y.-W., Tang, C.-L.: New existence and multiplicity results of homoclinic orbits for a class of second order Hamiltonian systems. Chaos Solitons Fractals 69, 151-159 (2014)

35. Yuan, R., Zhang, Z: Homoclinic solutions for a class of second order Hamiltonian systems. Results Math. 61, 195-208 (2012)

36. Zhang, Z., Xiang, T., Yuan, R.: Homoclinic solutions for subquadratic Hamiltonian systems without coercive conditions. Taiwan. J. Math. 18, 1089-1105 (2014)

37. Zhang, Z., Yuan, R.: Homoclinic solutions for a class of non-autonomous sub-quadratic second-order Hamiltonian systems. Nonlinear Anal. 71, 4125-4130 (2009)

\section{Submit your manuscript to a SpringerOpen ${ }^{\circ}$ journal and benefit from:}

- Convenient online submission

- Rigorous peer review

- Open access: articles freely available online

- High visibility within the field

- Retaining the copyright to your article

Submit your next manuscript at $\gg$ springeropen.com 